\title{
A Secure and Fast Image Encryption Scheme Based on Double Chaotic S-Boxes
}

\author{
Shenli Zhu ${ }^{1}{ }^{(D)}$, Guojun Wang ${ }^{2}$ and Congxu Zhu ${ }^{3, *}$ \\ 1 School of Computer Science, University of South China, Hengyang 421001, China \\ 2 School of Computer Science, Guangzhou University, Guangzhou 510006, China \\ 3 School of Computer Science and Engineering, Central South University, Changsha 410083, China \\ * Correspondence: zhucx@csu.edu.cn; Tel.: +86-0731-8882-7601
}

Received: 22 July 2019; Accepted: 12 August 2019; Published: 13 August 2019

\begin{abstract}
In order to improve the security and efficiency of image encryption systems comprehensively, a novel chaotic S-box based image encryption scheme is proposed. Firstly, a new compound chaotic system, Sine-Tent map, is proposed to widen the chaotic range and improve the chaotic performance of 1D discrete chaotic maps. As a result, the new compound chaotic system is more suitable for cryptosystem. Secondly, an efficient and simple method for generating S-boxes is proposed, which can greatly improve the efficiency of S-box production. Thirdly, a novel double S-box based image encryption algorithm is proposed. By introducing equivalent key sequences $\{\mathbf{r}, \mathbf{t}\}$ related with image ciphertext, the proposed cryptosystem can resist the four classical types of attacks, which is an advantage over other S-box based encryption schemes. Furthermore, it enhanced the resistance of the system to differential analysis attack by two rounds of forward and backward confusion-diffusion operation with double S-boxes. The simulation results and security analysis verify the effectiveness of the proposed scheme. The new scheme has obvious efficiency advantages, which means that it has better application potential in real-time image encryption.
\end{abstract}

Keywords: image encryption; compound chaotic system; S-box; image information entropy

\section{Introduction}

With the rapid development of network communication, image encryption has become a research hotspot in the field of image processing and information security. Since image information has the characteristics of large amounts of data, strong redundancy and high correlation between adjacent pixels, image encryption algorithms need not only high security, but also fast encryption speed. If the speed of encryption is low, the time consumed will be too long because of the large amount of image data. To encrypt multimedia information with large amounts of data, security and efficiency should be considered comprehensively [1-5]. Chaos-based cryptosystem just meets the need of image encryption, which leads to the research of chaos-based image encryption technology has been widely concerned by scholars. As for chaotic cryptography, a new chaotic system with better cryptographic performance deserves to be established. Some representative studies have contributed to this aspect [6-9]. How to generate key stream or encryption component with good performance is very important to the security of the image Cryptosystem [10-12]. How to design encryption algorithm is the core research content of the image Cryptosystem [13]. Cryptanalysis [14-16] is another important research direction of cryptography, which can help cryptographic designers improve the security of cryptographic algorithms.

Among many chaos-based image encryption algorithms, the permutation and diffusion (PD) pattern encryption algorithm proposed by Fridrich [17] is the most popular one. This image encryption algorithm structure consists of shuffling pixel positions and changing pixel values. The permutation 
(or shuffling, scrambling) process plays a role in confusing the relationship between the cipher image and plain image. The function of the diffusion process is to spread the change of one pixel value in the plain image to the whole range of the cipher image. Based on the basic confusion-diffusion architecture, researchers have proposed many novel concrete encryption strategies. In Ref. [18-24], authors proposed some different permutation strategies for image scrambling aiming at the confusion process. In Ref. [22,25-29], authors put forward some novel image diffusion algorithm. In Ref. [30-36], authors adopt new chaotic systems to improve the complexity and randomness of chaotic key streams. Some other cryptographic methods have also been tried by many researchers. For example, some cryptographic algorithms are based on bit-level permutation and diffusion [30], and some algorithms introduce the DNA coding mechanism [37], and some algorithms mainly use S-box to encrypt images [38-40]. However, some image encryption schemes exist as obvious security vulnerabilities. Thus, these image encryption schemes cannot resist some attacks, such as the chosen/known plaintext. In addition, some image encryption algorithms are inefficient, such as using bit-level image scrambling, DNA encoding mechanism, key related to plaintext Hash value [41,42], and the high-dimensional chaotic system $[43,44]$. Encryption algorithms with low efficiency are not suitable for some resource-constrained environments, such as mobile social network [45], sensor network communication environment [46] and searchable encryption [47]. Compared with high-dimensional continuous-time chaotic systems, low-dimensional discrete chaotic systems can generate chaotic sequences with higher efficiency. Moreover, some studies show that the complexity of discrete systems is higher than that of continuous systems [48-50].

Substitution-boxes (abbreviated as S-boxes) are important non-linear components in the block cipher system, which play an important role in the security of cryptosystems. Therefore, some image encryption systems based on chaos also use S-box. Majid Khan [51] employed multi-parameters chaotic systems in the construction of S-boxes that are applied to the encryption of images. The multi-parameters chaotic systems are hyper-chaotic systems. Moreover, the output trajectory points of the system need to be sampled, so the time cost of generating S-boxes in the encryption scheme is bound to be long. In addition, the S-box in the scheme is equivalent to the original key and is independent of the image content. Therefore, it is vulnerable to the chosen-plaintext attack. In order to resist the selective plaintext attack, some image encryption algorithms based on chaos introduce the mechanism of the key and plaintext association. Wang et al. [52] proposed a novel image encryption algorithm based on dynamic S-boxes constructed by chaos, in which a system up to 50 S-boxes need to be generated. It is time-consuming and unsuitable for real-time encryption. M.A. Murillo-Escobar et al. [53] proposed a color image encryption algorithm based on total plain image characteristics and 1D logistic map with optimized distribution. They have a diffusion process optimized by the modified chaotic sequence. In addition, the pseudorandom sequence for the encryption process is based on the total plain image characteristic and a 128 bits secret key, so the encryption algorithm can resist the powerful chosen-plaintext attack. Zhang et al. [54] proposed a plaintext-related image encryption algorithm based on chaos. The Zhang's system can also fight against the chosen-plaintext attacks due to using a plaintext-related key sequence. However, in order to make the final key related to the plaintext, the process of generating the final key in the above algorithms is complex. So far, most image and video encryption algorithms based on chaos mainly rely on the empirical security analysis. However, the recent study [55] has shown that the empirical safety analysis is not enough. A encryption algorithm passing the empirical safety tests is merely a necessary condition for security, but is not a sufficient criterion.

In order to improve the security and real-time performance of the image encryption algorithm, this paper presents a simple yet security image encryption algorithm based on chaotic S-boxes. The main goal of this paper is to improve the encryption efficiency of the encryption system on the premise of ensuring a certain level of security. The main innovations of this paper are as follows: (1) A new compound chaotic system, the Sine-Tent system (STS), is proposed. The compound system has wider chaotic range and better chaotic performance than any of the original systems, so it is more suitable 
for cryptographic applications. (2) A simple and effective S-box construction method based on the new compound chaotic system is proposed, which can speed up the generation of S-boxes. (3) A double S-boxes based image encryption algorithm is designed. Double S-boxes can not only meet the security requirements of the system, but also make the time cost much lower than multiple S-boxes. The algorithm makes the parameters of the permutation and diffusion process interrelated and related with image ciphertext so that the encryption algorithm can resist chosen-ciphertext attack. Additionally, two rounds of forward and backward confusion-diffusion operation enhances the resistance of the system to the differential analysis attack.

The rest of this paper is organized as follows. Section 2 introduces the new Sine-Tent system (STS) model. Section 3 describes the simple and effective S-box construction method based on the Sine-Tent system. Section 4 describes the new double S-boxes based image encryption algorithm. Section 5 presents the results of experiments and analysis of the proposed scheme. Finally, some concluding remarks are given in Section 6.

\section{The Proposed New Chaotic System}

1D discrete chaotic systems have many advantages in image encryption because of their simple structures. In this section, we firstly review two 1D chaotic maps: The Sine and Tent maps. They will be used for constructing our new chaotic system. Then, a new discrete compound chaotic system is proposed to solve the problems existing in the Sine and Tent maps.

\subsection{Sine Chaotic Map}

The Sine map is one of the famous 1D chaotic maps. It is a simple dynamical system with complex chaotic behavior similar to the Logistic map. The mathematical model of the Sine map can be expressed as

$$
x(n+1)=\mu / 4 \times \sin (\pi \times x(n))
$$

where $\mu$ is the system parameter in the range of $(0,4], x(0)$ is the initial state value of the system and $\{x(n), n=1,2, \ldots\}$ is the output sequence of state values. To observe the chaotic behaviors of the Sine map, its Lyapunov Exponent and bifurcation diagram are presented in Figure 1a,b.

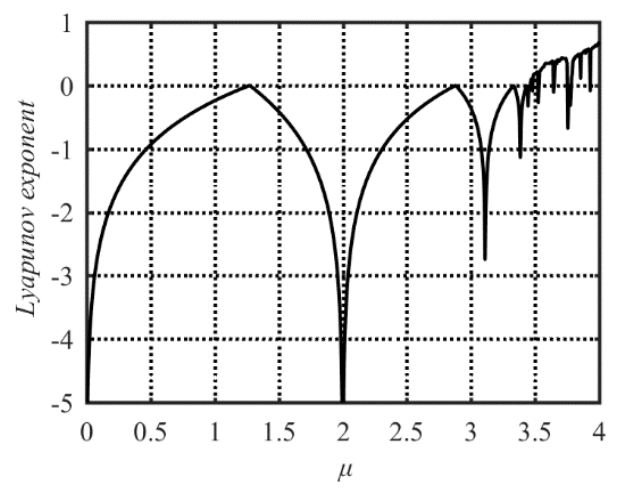

(a)

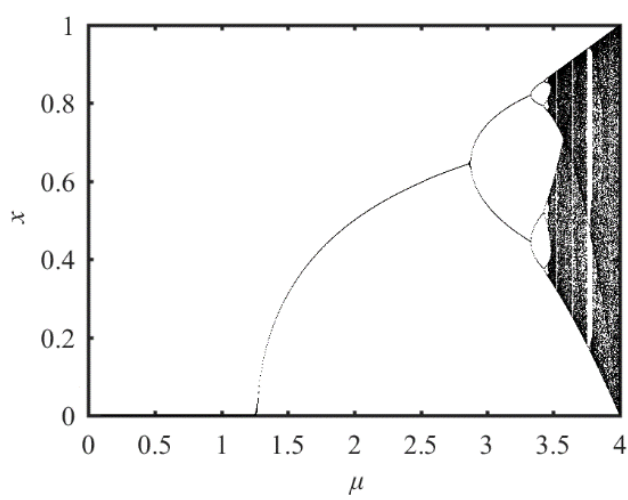

(b)

Figure 1. Lyapunov Exponent and bifurcation diagram of the Sine map. (a) Lyapunov Exponent diagram; (b) bifurcation diagram.

As is well known, for a dynamical system, a positive Lyapunov Exponent means chaotic behavior occurs in the dynamical system. So, from Figure 1a, one can see that only when the parameter $\mu \geq 3.57$ can chaotic behavior occur in the Sine map. The bifurcation diagram depicts the possible state values of the system under each parameter. Corresponding to a value of system parameter, if there are infinite state values, the system with the parameter has chaotic behavior. Corresponding to a value of system parameter, if only one or a limited number of state values output, the system with the parameter does 
not have chaotic behavior. In the bifurcation diagram shown in Figure $1 b$, the areas of $\mu$ with dense points shows its good chaotic behavior and the areas of $\mu$ with the solid line represents its non-chaotic property. There are two problems in the Sine map. First, the range of system parameters corresponding to chaotic phenomena is limited only within the range of $[3.57,4]$. Even within this range, there are some parameters which make the Sine map have no chaotic behaviors. This is verified by its Lyapunov Exponent diagram and the blank zone in its bifurcation diagram. Second, when the system parameter value is less than four, the state values of the system output sequence are distributed in a narrower range than the $[0,1]$ interval. Only when the system parameter value is four, the state values of the system output sequence are distributed in the whole $[0,1]$ range. It shows the nonuniform distribution in the range of $[0,1]$. These two problems reduce the application value of the Sine map.

\subsection{Tent Chaotic Map}

The name "Tent map" comes from its bifurcation diagram, which has the tent-like shape. Its mathematical model can be expressed as

$$
x(n+1)= \begin{cases}\mu / 2 \times x(n) & x(n)<0.5 \\ \mu / 2 \times(1-x(n)) & x(n) \geq 0.5\end{cases}
$$

where $\mu$ is the system parameter in the range of $(0,4]$.

Its chaotic property is shown in the Lyapunov Exponent analysis in Figure 2a and bifurcation analysis in Figure $2 \mathrm{~b}$. Both analysis results indicate that its parameter value range with chaotic behavior is $2 \leq \mu \leq 4$. The Tent map has the same problems as the Sine map: The small parameter value range with chaotic behavior and the nonuniform distribution of the output state values.

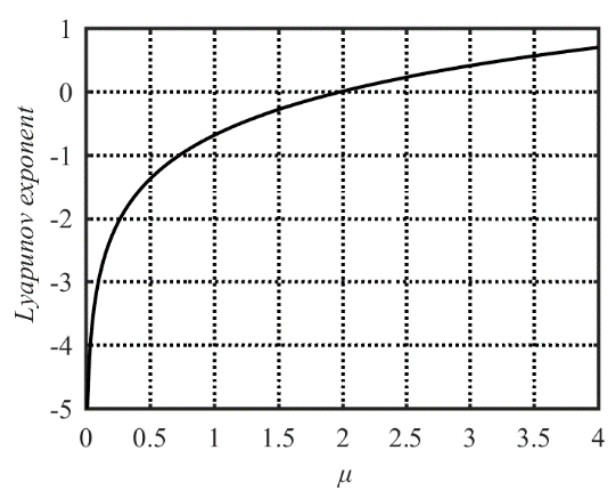

(a)

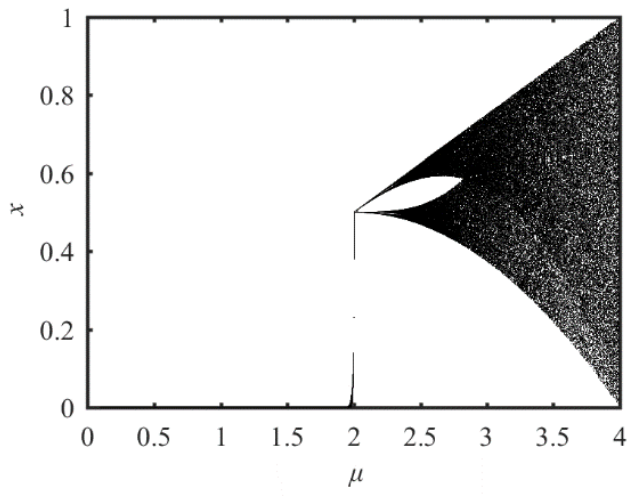

(b)

Figure 2. Lyapunov Exponent and bifurcation diagram of the Tent map. (a) Lyapunov Exponent diagram; (b) bifurcation diagram.

\subsection{The Sine-Tent System}

We put forward a new compound system by combining the Sine and Tent maps and called the new system the Sine-Tent system (STS). Its mathematical model is as follows:

$$
x(n+1)= \begin{cases}(4-\mu) / 4 \times \sin (\pi \times x(n))+\mu / 2 \times x(n) & x(n)<0.5 \\ (4-\mu) / 4 \times \sin (\pi \times x(n))+\mu / 2 \times(1-x(n)) & x(n) \geq 0.5\end{cases}
$$

where $\mu$ is the system parameter in the range of $[0,4]$. When $\mu=0$, Equation (3) degenerates to the Sine map, while $\mu=4$, Equation (3) degenerates to the Tent map. Therefore, both the Sine map and Tent map can be regarded as special cases of the Sine-Tent system.

The Lyapunov Exponent and bifurcation diagram of the STS are shown in Figure 3a,b, respectively. From Figure 3 one can see that its parameter value range with chaotic behavior is $\mu \in[0,4]$, which is 
much larger than those of the Sine or Tent maps. Its output sequences uniformly distribute within $[0,1]$ (see Figure 3b). Hence, the STS has better chaotic performance than the Sine and Tent maps.

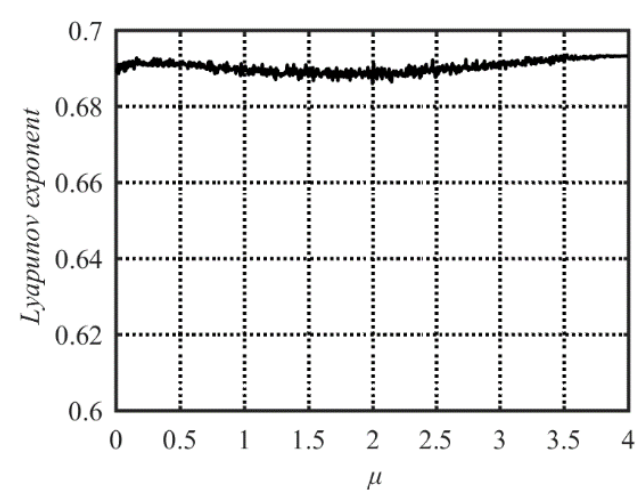

(a)

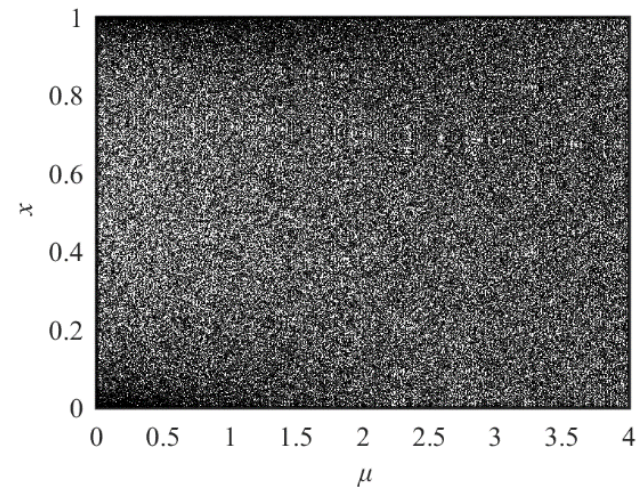

(b)

Figure 3. Lyapunov Exponent and bifurcation diagram of the Sine-Tent map. (a) Lyapunov Exponent diagram; (b) bifurcation diagram.

The new compound system has at least three advantages compared with the Sine and Tent maps. First, the output sequences of the new compound system spread out in the entire value range between zero and one. Second, the proposed Sine-Tent system has a wider chaotic range. The Lyapunov Exponents of the Sine-Tent system is positive in the entire range of $0 \leq \mu \leq 4$. However, the Sine map and Tent map have positive values of Lyapunov Exponents only within much smaller ranges. Thirdly, we know that a larger Lyapunov Exponent means stronger chaotic properties. From the Lyapunov Exponent diagrams, one can see that the new system has larger Lyapunov Exponents (Lyapunov Exponents is always close to 0.7 ) in the whole parameter range of [0, 4], while the Sine and Tent maps have large Lyapunov Exponents only when the parameter is close to four. Therefore, the chaotic characteristic of the new system is stronger, and it always maintains the invariable excellent chaotic performance in the entire parameter range of $0 \leq \mu \leq 4$. These advantages guarantee that the proposed Sine-Tent system is more suitable for information security applications such as image encryption.

\section{An Efficient New Method for Generating S-Boxes}

In Ref. [56], Belazi et al. proposed a simple yet efficient S-box generating method based on the chaotic sine map, in which a prime number $p$ and a one to one map from the real number interval $(0,1)$ to the integer set $\{0,1,2, \ldots, 255\}$ need to be found. In this section, we present a simpler approach for designing S-boxes using the chaotic Sine-Tent map. The new method takes advantage of the excellent chaotic characteristics of the Sine-Tent map. The detailed steps of generating S-boxes are given below.

Step 1: Set parameter $d$ as an odd positive integer and $d>0, d$ can be used as a secret key.

Step 2: Let $\mathbf{T 1}=1: 256$, then we obtain an array $\mathbf{T} 1$ which contains 256 distinct integers in the range of $[1,256]$.

Step 3: Based on $\mathbf{T} 1$ and $d$ to obtain a new array $\mathbf{T}$ by Equation (4)

$$
\mathrm{T}(i)=\bmod (d \times \mathrm{T} 1(i), 256), i=1,2, \ldots, 256
$$

The new array $\mathbf{T}_{1 \times 256}$ will contain 256 distinct integers in the range of $[0,255]$. As long as $d$ is a finite odd integer and $\mathrm{T} 1(i) \neq \mathrm{T} 1(j)$ if $i \neq j$, then $\mathrm{T}(i) \neq \mathrm{T}(j)$ if $i \neq j$. This conclusion is true and can be proved by experimental tests.

Step 4: Set the parameters $\mu$, initial state value $x_{0}$ of the Sine-Tent map, and an integer $N_{0}>0$. Iterate Sine-Tent map $\left(N_{0}+256\right)$ times to generate a chaotic sequence of length $\left(N_{0}+256\right)$. Discard the first $N_{0}$ elements of the original chaotic sequence, then we can obtain a new chaotic sequence of length 256 , which is represented by $\mathbf{X}$. 
Step 5: Sort the chaotic sequence $\mathbf{X}$, then we can get a position index array $\mathbf{J}=\{\mathrm{J}(1), \mathrm{J}(2), \ldots$, $\mathrm{J}(256)\}, \mathrm{J}(i) \in\{1,2, \ldots, 256\}$. As a result of the non-periodicity of the chaotic sequence, it will inevitably lead to that $\mathrm{J}(i) \neq \mathrm{J}(j)$ as long as $i \neq j$.

Step 6: Calculate the 1D array $\mathbf{S}$ as follows:

$$
\mathrm{S}(i)=\mathrm{T}(\mathrm{J}(i)), i=1,2, \ldots, 256
$$

Step 7: Transform the 1D array $\mathbf{S}_{1 \times 256}$ into a $2 \mathrm{D}$ matrix $\mathbf{S}_{16 \times 16}$, and this is the proposed S-box.

By the above method, the length of chaotic sequences to be used in constructing a $16 \times 16$ sized S-box is only 256. Therefore, the time cost of this method is very low. In our experiments, double S-boxes are generated by the above S-box generation algorithm. The initial condition $x_{0}$, system parameter $\mu$ of the Sine-Tent map and the parameters $\left\{d, N_{0}\right\}$ for the S-box generation are set as $\left\{x_{10}=0.21, \mu_{1}=0.399, d_{1}=43, N_{0}=500\right\}$ and $\left\{x_{20}=0.27, \mu_{2}=3.999, d_{2}=241, N_{0}=500\right\}$ for S-box S1 and S2, respectively. The generated double S-boxes are shown in Tables 1 and 2, which are used in our proposed image encryption algorithm.

Table 1. The chaotic S-box S1 generated with parameters $\left\{x_{10}=0.21, \mu_{1}=0.399, d_{1}=43, N_{0}=500\right\}$.

\begin{tabular}{ccccccccccccccccc}
\hline S-box & $\mathbf{c 1}$ & $\mathbf{c 2}$ & $\mathbf{c 3}$ & $\mathbf{c 4}$ & $\mathbf{c 5}$ & $\mathbf{c 6}$ & $\mathbf{c 7}$ & $\mathbf{c 8}$ & $\mathbf{c 9}$ & $\mathbf{c 1 0}$ & $\mathbf{c 1 1}$ & $\mathbf{c 1 2}$ & $\mathbf{c 1 3}$ & $\mathbf{c 1 4}$ & $\mathbf{c 1 5}$ & $\mathbf{c 1 6}$ \\
\hline r1 & 27 & 4 & 47 & 58 & 146 & 86 & 137 & 215 & 61 & 68 & 129 & 80 & 131 & 214 & 97 & 119 \\
r2 & 168 & 210 & 253 & 91 & 219 & 30 & 112 & 63 & 52 & 188 & 73 & 139 & 55 & 16 & 158 & 204 \\
r3 & 124 & 71 & 21 & 45 & 169 & 32 & 208 & 121 & 198 & 179 & 246 & 8 & 175 & 194 & 35 & 5 \\
r4 & 70 & 3 & 114 & 42 & 205 & 89 & 101 & 159 & 173 & 127 & 75 & 235 & 118 & 243 & 143 & 141 \\
r5 & 147 & 13 & 196 & 163 & 11 & 62 & 134 & 76 & 191 & 133 & 132 & 145 & 33 & 43 & 120 & 31 \\
r6 & 17 & 156 & 245 & 186 & 25 & 237 & 88 & 161 & 0 & 83 & 87 & 72 & 116 & 150 & 255 & 226 \\
r7 & 138 & 74 & 46 & 34 & 136 & 99 & 12 & 218 & 110 & 195 & 105 & 57 & 172 & 65 & 2 & 216 \\
r8 & 211 & 184 & 19 & 20 & 84 & 242 & 85 & 98 & 189 & 22 & 24 & 185 & 166 & 109 & 15 & 217 \\
r9 & 167 & 48 & 56 & 78 & 90 & 59 & 36 & 244 & 6 & 107 & 142 & 180 & 23 & 238 & 106 & 7 \\
r10 & 28 & 247 & 199 & 201 & 40 & 250 & 206 & 183 & 223 & 200 & 29 & 67 & 128 & 126 & 10 & 241 \\
r11 & 113 & 233 & 207 & 140 & 152 & 135 & 122 & 174 & 228 & 151 & 102 & 148 & 79 & 176 & 49 & 95 \\
r12 & 190 & 103 & 92 & 39 & 64 & 1 & 171 & 220 & 212 & 51 & 221 & 130 & 249 & 170 & 164 & 230 \\
r13 & 60 & 162 & 117 & 154 & 157 & 160 & 229 & 187 & 100 & 26 & 37 & 155 & 225 & 222 & 232 & 104 \\
r14 & 181 & 224 & 53 & 18 & 108 & 96 & 66 & 38 & 248 & 182 & 178 & 251 & 165 & 231 & 202 & 81 \\
r15 & 50 & 93 & 149 & 9 & 239 & 192 & 209 & 82 & 115 & 236 & 44 & 144 & 69 & 111 & 153 & 125 \\
r16 & 254 & 41 & 227 & 213 & 193 & 14 & 77 & 197 & 54 & 123 & 203 & 177 & 94 & 252 & 234 & 240 \\
\hline
\end{tabular}

Table 2. The chaotic S-box S2 generated with parameters $\left\{x_{20}=0.27, \mu_{2}=3.999, d_{2}=241, N_{0}=500\right\}$.

\begin{tabular}{ccccccccccccccccc}
\hline S-box & $\mathbf{c 1}$ & $\mathbf{c 2}$ & $\mathbf{c 3}$ & $\mathbf{c 4}$ & $\mathbf{c 5}$ & $\mathbf{c 6}$ & $\mathbf{c 7}$ & $\mathbf{c 8}$ & $\mathbf{c 9}$ & $\mathbf{c 1 0}$ & $\mathbf{c 1 1}$ & $\mathbf{c 1 2}$ & $\mathbf{c 1 3}$ & $\mathbf{c 1 4}$ & $\mathbf{c 1 5}$ & $\mathbf{c 1 6}$ \\
\hline r1 & 75 & 140 & 59 & 156 & 233 & 234 & 149 & 214 & 126 & 105 & 134 & 228 & 101 & 84 & 111 & 35 \\
r2 & 113 & 241 & 53 & 202 & 17 & 96 & 93 & 168 & 172 & 82 & 78 & 203 & 159 & 182 & 249 & 118 \\
r3 & 115 & 68 & 195 & 107 & 189 & 104 & 165 & 80 & 39 & 94 & 150 & 254 & 199 & 183 & 157 & 74 \\
r4 & 52 & 210 & 55 & 200 & 229 & 48 & 132 & 163 & 219 & 201 & 117 & 146 & 153 & 43 & 71 & 230 \\
r5 & 60 & 70 & 103 & 211 & 95 & 92 & 36 & 12 & 81 & 133 & 46 & 176 & 209 & 251 & 237 & 186 \\
r6 & 98 & 136 & 20 & 44 & 178 & 185 & 177 & 19 & 137 & 50 & 21 & 206 & 65 & 192 & 129 & 79 \\
r7 & 240 & 7 & 121 & 38 & 27 & 196 & 25 & 167 & 89 & 72 & 162 & 221 & 148 & 147 & 24 & 223 \\
r8 & 100 & 47 & 248 & 164 & 34 & 29 & 73 & 69 & 245 & 1 & 10 & 191 & 216 & 26 & 204 & 18 \\
r9 & 37 & 15 & 32 & 108 & 9 & 160 & 139 & 220 & 238 & 232 & 58 & 161 & 109 & 6 & 169 & 62 \\
r10 & 45 & 3 & 0 & 180 & 114 & 120 & 246 & 250 & 33 & 194 & 198 & 13 & 158 & 31 & 66 & 155 \\
r11 & 83 & 125 & 244 & 51 & 212 & 97 & 91 & 99 & 77 & 138 & 173 & 243 & 253 & 102 & 123 & 166 \\
r12 & 225 & 208 & 110 & 40 & 222 & 87 & 218 & 197 & 170 & 184 & 124 & 131 & 4 & 112 & 179 & 255 \\
r13 & 85 & 64 & 193 & 88 & 56 & 16 & 236 & 207 & 181 & 144 & 231 & 239 & 152 & 135 & 122 & 67 \\
r14 & 151 & 171 & 42 & 154 & 142 & 247 & 28 & 41 & 14 & 252 & 224 & 188 & 54 & 175 & 217 & 130 \\
r15 & 22 & 215 & 49 & 5 & 141 & 11 & 2 & 127 & 145 & 86 & 116 & 213 & 205 & 63 & 242 & 128 \\
r16 & 30 & 226 & 227 & 106 & 187 & 23 & 174 & 190 & 143 & 8 & 76 & 61 & 235 & 119 & 57 & 90 \\
\hline
\end{tabular}

In the first row of Table 1, c1, c2, . , c16 denotes the column numbers of the S-box. Additionally, in the first column of Table 1, r1, r2, .. , r16 denotes the row numbers of the S-box. 
To determine the randomness of proposed S-box method, the statistical test suite (version 2.1.1), proposed by the National Institute of Standards and Technology (NIST) NIST-800-22 is introduced. The NIST-800-22 test results are listed in Table 3. We find that the 12 tests successfully passed. Moreover, the Random Excursions Test, Random Excursions Variant Test, and Universal Statistical Test were not applicable for the proposed S-box. This is because the sequence generated by an S-box only consists of 2048 bits. However, the Random Excursions Test and Random Excursions Variant Test require a long sequence consisting of a minimum of 1,000,000 bits, and the Universal Statistical Test also requires a long sequence consisting of a minimum of 387,840 bits.

Table 3. NIST-800-22 test results of the obtained S-box.

\begin{tabular}{ccc}
\hline NIST-800-22 Tests & $p$-Value & Result \\
\hline Frequency Test & 1.00000 & SUCCESS \\
Block Frequency Test & 0.320250 & SUCCESS \\
Cumulative Sums Test & 0.536610 & SUCCESS \\
Runs Test & 0.894524 & SUCCESS \\
Longest Run of Ones Test & 1.0000 & SUCCESS \\
Rank Test & 0.481248 & SUCCESS \\
Discrete Fourier Transform Test & 0.807748 & SUCCESS \\
Nonperiodic Template Matchings Test & 0.861831 & SUCCESS \\
Overlapping Template Matchings Test & 0.282761 & SUCCESS \\
Approximate Entropy Test & 0.011732 & SUCCESS \\
Serial Test & 0.239176 & SUCCESS \\
Linear Complexity Test & 0.203697 & SUCCESS \\
Random Excursions Test & $\backslash$ & TESTNOTAPPLICABLE \\
Random Excursions Variant Test & $\backslash$ & TESTNOTAPPLICABLE \\
Universal Statistical Test & $\backslash$ & TESTNOTAPPLICABLE \\
\hline
\end{tabular}

\section{The Proposed S-Box based Encryption Scheme}

\subsection{Cryptanalysis of an S-Box Based Encryption Algorithm}

In Ref. [57], Çavuşoğlu et al. proposed an image encryption scheme by using the S-box generated with a novel hyper-chaotic system. The sketch of the encryption scheme is shown in Figure 4.

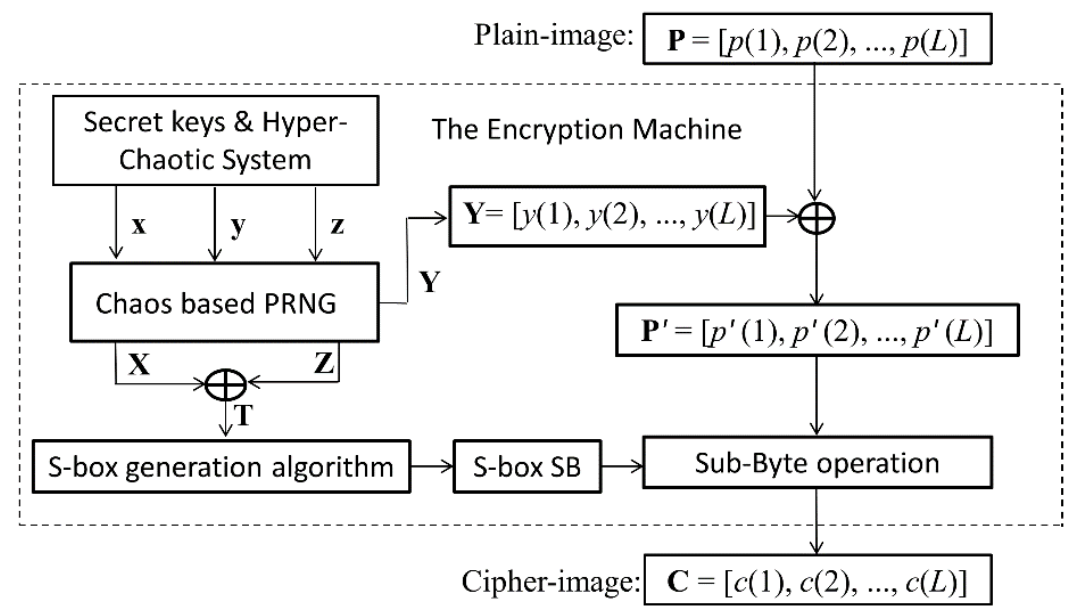

Figure 4. Sketch of the original encryption algorithm.

Suppose the input pixel value array of the plain image is $\mathbf{P}=[p(1), p(2), \ldots, p(L)]$. The output pixel value array of the cipher image is $\mathbf{C}=[c(1), c(2), \ldots, c(L)]$. The encryption steps can be described in detail below. 
Step 1: Generate three real value chaotic sequences $\mathbf{x}, \mathbf{y}$, and $\mathbf{z}$ by using a hyper-chaotic system with given parameters and initial state values as secret keys.

Step 2: Transform the three real value sequences $\mathbf{x}, \mathbf{y}$ and $\mathbf{z}$ into three integer sequences $\mathbf{X}, \mathbf{Y}$ and $\mathbf{Z}$ by the chaos-based pseudo random number generator (PRNG). Each element in $\mathbf{X}, \mathbf{Y}$ and $\mathbf{Z}$ is an 8 -bit integer and its decimal number is in the range of [0, 255].

Step 3: The S-box, denoted as $\mathbf{S}=[s(j, k)]$, is created by using sequences $\mathbf{X}, \mathbf{Z}$ and a novel S-box generation algorithm. Where, $s(j, k) \in\{0,1, \ldots, 255\}, j=1,2, \ldots, 16, k=1,2, \ldots, 16$.

Step 4: The intermediate cipher image array $\mathbf{P}^{\prime}=\left[p^{\prime}(1), p^{\prime}(2), \ldots, p^{\prime}(L)\right]$ is generated by using sequences $\mathbf{Y}=[y(1), y(2), \ldots, y(L)]$ as

$$
p^{\prime}(i)=y(i) \bigoplus p(i), i=1,2, \ldots, L
$$

where $\oplus$ denotes bitwise XOR. The decryption operation corresponding to Equation (6a) can be expressed as Equation (6b):

$$
p(i)=y(i) \bigoplus p^{\prime}(i), i=1,2, \ldots, L
$$

Step 5: Perform sub-byte operation on $\mathbf{P}^{\prime}$ with the $16 \times 16$ sized S-box $\mathbf{S}$, and obtain the cipher image array $\mathbf{C}=[c(1), c(2), \ldots, c(L)]$.

Here, the sub-byte operation is a process in which each pixel value in the image is substituted with an element value in the S-box. The sub-byte operation can be implemented by defining a function. For example, the function sub_byte[S, $p]$ can find a substitute to $p$ from the S-box $\mathbf{S}$. Let $q=\operatorname{sub} \_$byte[S,$\left.p\right]$, the algorithm of the function sub_byte[S, $\left.p\right]$ can be described as Algorithm 1 . For example, if $p=55=(00110111)_{2}$, then $j=(0011)_{2}+1=4, k=(0111)_{2}+1=8$. Consequently, $q=$ sub_byte $[\mathbf{S}, p]=$ sub_byte $[\mathbf{S}, 55]=s(j, k)=s(4,8)$.

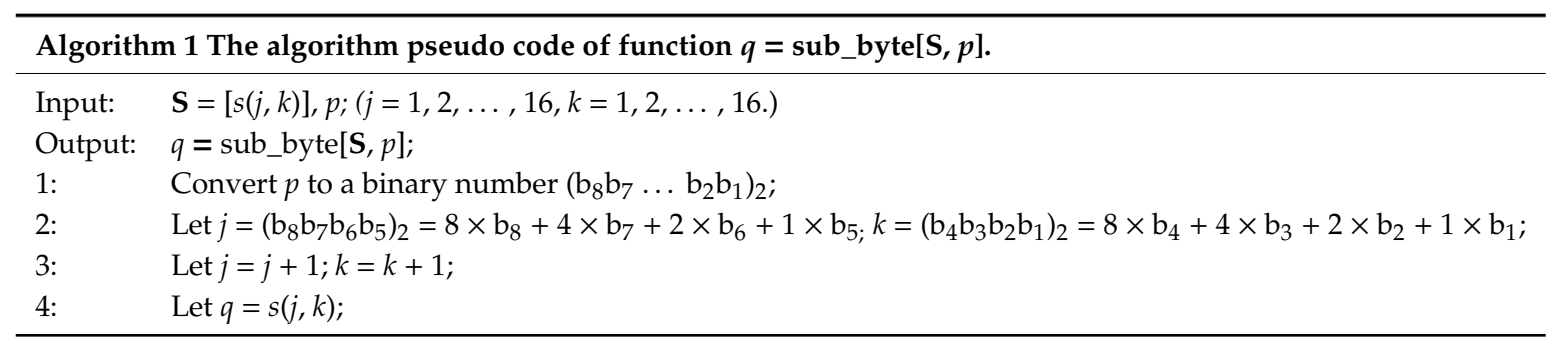

Therefore, Step 5 can be expressed by the following general form:

$$
c(i)=\operatorname{sub} \_ \text {byte}\left[\mathbf{S}, p^{\prime}(i)\right], i=1,2, \ldots, L
$$

The decryption operation corresponding to Equation (7a) can be expressed as Equation (7b):

$$
\left.p^{\prime}(i)=\text { sub_byte_1[S, } c(i)\right], i=1,2, \ldots, L
$$

where, function sub_byte_1[?, $\cdot]$ is the inverse operation of the function sub_byte[., $\cdot]$.

The above S-box based encryption algorithm has the following potential defects:

(1) The chaotic sequence $\mathbf{Y}$ and S-box is actually the equivalent of the secret keys, which are not related with the image to be encrypted.

(2) The algorithm has no diffusion effect. While one pixel is changed in the plain image, there is only one changed pixel in the cipher image.

(3) The sequence $Y$ and S-box are separated in the bitwise XOR process and Sub-Byte process, and the bitwise XOR process unrelated to the Sub-Byte process.

Based on the above analysis, we find that the above encryption scheme cannot resist the chosen-plaintext attack. Suppose the target cipher image to be recovered is $\mathbf{C}=[c(1), c(2), \ldots, c(L)]$, we 
can launch chosen-plaintext attack on the above encryption scheme to recover its corresponding plain image $\mathbf{P}=[p(1), p(2), \ldots, p(L)]$. The simplest attacking algorithm can be described as Algorithm 2 .

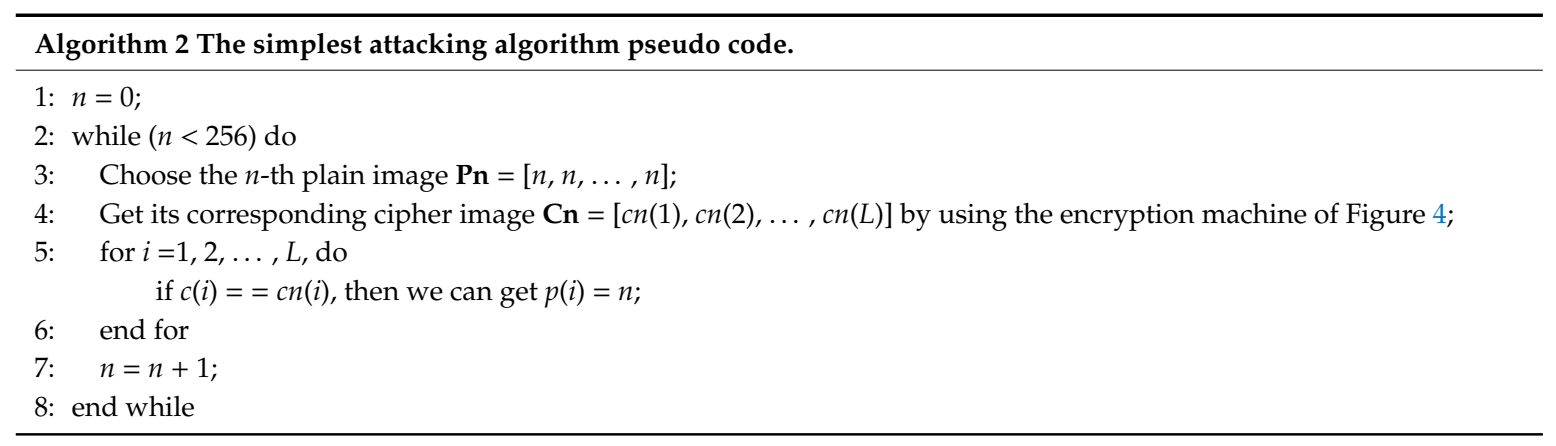

This simplest attack method with Algorithm 2 requires 256 selected plaintext images. However, a more efficient chosen-plaintext method only needs to select two plain images. For details, readers can refer to Ref. [58].

\subsection{The Novel Double S-Boxes Based Image Encryption Algorithm}

To eliminate the security defects that exist in some S-box based encryption algorithms, a novel double S-boxes based image encryption algorithm is proposed. The main innovations of the new scheme lie in the following three aspects: Firstly, the new Sine-Tent compound chaotic system is used to generate double S-boxes, which are used in the two rounds of the encryption process of the new scheme. Secondly, the first S-box is used to realize pixel confusion and substitution simultaneously. Thirdly, two rounds of the encryption process are correlated and the diffusion mechanism is introduced. The main steps of the novel double S-boxes based image encryption algorithm is described as follows:

Step 1: Input the secret parameters $\left\{x_{10}, \mu_{1}, d_{1}, x_{20}, \mu_{2}, d_{2}, r_{0}, t_{0}, m\right\}$ and the plain image PI with the size of $M \times N$. PI is reshaped to a 1D pixel array $\mathbf{P}=[p(1), p(2), \ldots, p(L)]$, where $L=M \times N$.

Step 2: Generate the first S-box $\mathbf{S} 1$ by using the new S-box generation algorithm with parameters $\left\{x_{10}, \mu_{1}, d_{1}\right\}$.

Step 3: Generate the second S-box S2 by using the new S-box generation algorithm with parameters $\left\{x_{20}, \mu_{2}, d_{2}\right\}$.

Step 4: Perform the first round of encryption on array $\mathbf{P}$ with the first S-box $\mathbf{S 1}$, and obtain the temporary cipher image pixel array $\mathbf{B}=[b(1), b(2), \ldots, b(L)]$ as

$$
\begin{gathered}
\left\{\begin{array}{l}
j=\bmod (1+m, L)+1 ; \\
r=r_{0} ; \\
b(1)=\bmod \left(\operatorname{sub} \_b y t e[\mathbf{S} 1, p(j)]+r, 256\right) .
\end{array}\right. \\
\left\{\begin{array}{l}
j=\bmod (i+m, L)+1 ; \\
r=\bmod (b(i-1)+r, 256) ; \\
b(i)=\bmod \left(\operatorname{sub} \_ \text {byte }[\mathbf{S} 1, p(j)]+r+b(i-1), 256\right) .
\end{array}\right.
\end{gathered}
$$

where, sub_byte[S1, $x$ ] denotes byte substitution for $x$ using S-box S1. The first round of encryption is the forward confusion-diffusion operation, in which permutation and diffusion are implemented simultaneously by introducing the location index $j$.

Step 5: Perform the second round of encryption on array B with the second S-box S2, and obtain the final cipher image pixel array $\mathbf{C}=[c(1), c(2), \ldots, c(L)]$ as

$$
\left\{\begin{array}{l}
t=t_{0} \\
c(L)=\operatorname{sub} \_ \text {byte }[\mathbf{S} 2, \bmod (b(L)+t, 256)] .
\end{array} \text { for } i=L\right.
$$




$$
\left\{\begin{array}{l}
t=\bmod (c(i+1)+t, 256) ; \\
c(i)=\operatorname{sub} \_ \text {byte}[\mathbf{S} 2, \bmod (b(i)+c(i+1)+t, 256)] .
\end{array} \text { for } i=L-1, L-2, \ldots, 1\right.
$$

where, sub_byte[S2, $x]$ denotes byte substitution for $x$ using S-box S2. The second round of encryption is the backward diffusion operation.

Step 6: Transform the $1 \mathrm{D}$ vector $\mathrm{C}$ into a 2D matrix with size of $M \times N$, then the cipher image $\mathrm{CI}$ is obtained.

The decryption process is the inverse operation of the encryption process. To recover the plain image $\mathbf{P}$ from the cipher image $\mathbf{C I}$, the operating steps are as follows.

Step 1: Input the secret parameters $\left\{x_{10}, \mu_{1}, d_{1}, x_{20}, \mu_{2}, d_{2}, r_{0}, t_{0}, m\right\}$ and the cipher image $\mathbf{C I}$ with the size of $M \times N$, and $\mathbf{C I}$ is reshaped to a 1D pixel array $\mathbf{C}=[c(1), c(2), \ldots, c(L)]$, where $L=M \times N$.

Step 2: Generate the first S-box S1. The operation is exactly the same as Step 2 of the encryption process.

Step 3: Generate the second S-box S2. The operation is exactly the same as Step 3 of the encryption process.

Step 4: Recover the intermediate cipher image pixel array $\mathbf{B}=[b(1), b(2), \ldots, b(L)]$ as

$$
\begin{gathered}
\left\{\begin{array}{l}
t=t_{0} ; \\
b(L)=\bmod \left(s u b \_b y t e \_1(\mathbf{S} 2, c(L))-t+256,256\right) .
\end{array} \text { for } i=L .\right. \\
\left\{\begin{array}{l}
t=\bmod (c(i+1)+t, 256) \\
b(i)=\bmod \left(s u b \_b y t e \_1(\mathbf{S} 2, c(i))-t-c(i+1)+256,256\right)
\end{array} \text { for } i=L-1, L-2, \ldots, 1\right.
\end{gathered}
$$

where, sub_byte_1[S2, $\cdot]$ denotes the inverse operation of sub_byte[S2, $\cdot]$ using S-box S2.

Step 5: Recover the original plain image pixel array $\mathbf{P}=[p(1), p(2), \ldots, p(L)]$ as

$$
\begin{gathered}
\left\{\begin{array}{l}
j=\bmod (1+m, L)+1 ; \\
r=r 0 ; \\
p(j)=\text { sub_byte_1 }(\mathbf{S} 1, \bmod (b(1)-r+256,256)) .
\end{array}\right. \\
\left\{\begin{array}{l}
j=\bmod (i+m, L)+1 ; \\
r=\bmod (b(i-1)+r, 256) ; \\
p(j)=\operatorname{sub} \_ \text {byte_1 }(\mathbf{S} 1, \bmod (b(i)-b(i-1)-r+256,256)) .
\end{array}\right.
\end{gathered}
$$

where, sub_byte_1[S1, $]$ denotes the inverse operation of sub_byte[S1, $\cdot]$ using S-box S1.

Step 6: Transform $\mathbf{P}$ into an $M \times N$ matrix, then the decrypted image PI is obtained.

\section{Experimental Results and Security Analyses}

To examine the security and efficiency of the proposed cryptosystem, we carry out some simulation experiments. All the algorithms are implemented with MATLAB R2016b run on a Microsoft Windows 7 operating system. The hardware environment is a PC with $3.3 \mathrm{GHz} \mathrm{CPU}$, and $4 \mathrm{~GB}$ memory. Without losing generality, we adopted the public test images come from the USC-SIPI Image Database. Test images are 8-bit grayscale images with a size of $256 \times 256$, such as Lena, Baboon, Pepper. The all-black and all-white images are also used in the simulation experiments. The secret keys $\left\{x_{10}, \mu_{1}, d_{1}, x_{20}, \mu_{2}\right.$, $\left.d_{2}, r_{0}, t_{0}, m\right\}$ are set as $\{0.21,0.399,43,0.27,3.999,241,98,200,129\}$.

\subsection{Experimental Results}

The original plain images and their corresponding cipher-images are shown in Figures 5 and 6, respectively. While the decrypted images are identical to the corresponding original ones. As can be seen, the cipher-images are completely disordered and unrecognizable. Therefore, our proposed algorithm has a good encryption effect. 


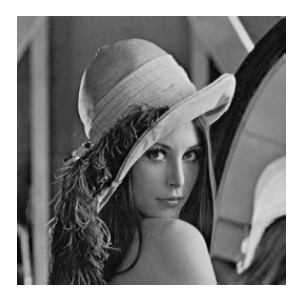

(a)

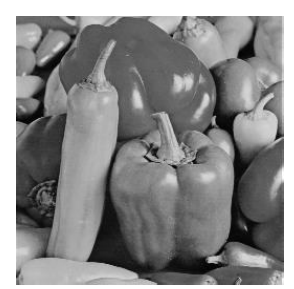

(b)

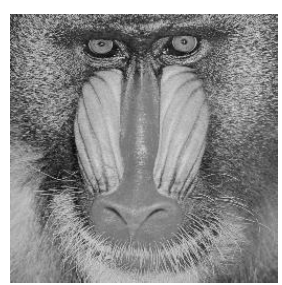

(c)

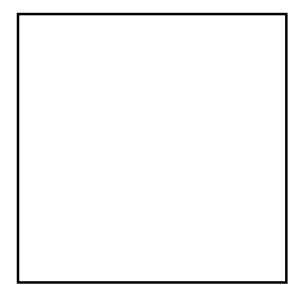

(d)

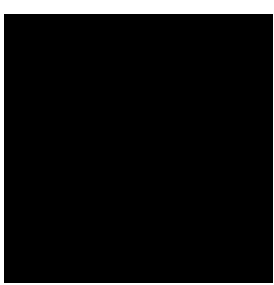

(e)

Figure 5. Original plain images. (a) The Lena plain image; (b) the Peppers plain image; (c) the Baboon plain image; (d) the all-white image; (e) the all-black image.

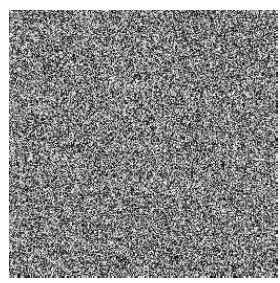

(a)

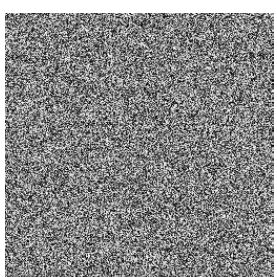

(b)

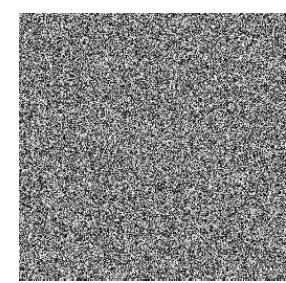

(c)

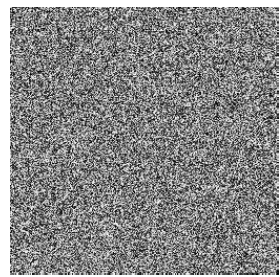

(d)

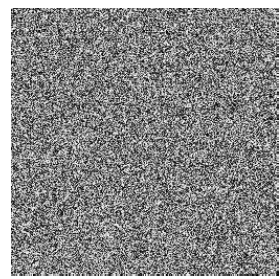

(e)

Figure 6. Encrypted cipher images. (a) The Lena cipher image; (b) the Peppers cipher image; (c) the Baboon cipher image; (d) the all-white cipher-image; (e) the all-black cipher image.

\subsection{Key Space Analyses}

A secure encryption scheme should have a large key space so as to resist brute-force attack. In our proposed encryption scheme, the secret keys include $\left\{x_{10}, \mu_{1}, d_{1}, x_{20}, \mu_{2}, d_{2}, r_{0}, t_{0}, m\right\}$. Among them, $\left\{x_{10}, \mu_{1}, x_{20}, \mu_{2}\right\}$ are four double-precision real numbers, each of them can reach the accuracy of 15 decimal places. $d_{1}$ and $d_{2}$ are two odd integers, each of them can have $10^{4}$ different values. $r_{0}$ and $t_{0}$ are two integers, each of them has 255 different values. $m$ is an integer range from 1 to $L$, where $L=65536$. So, the key space of our proposed encryption scheme is $\left(10^{15 \times 4+4 \times 2}\right) \times 255 \times 255 \times 65536 \approx 2^{258}$, which is a key equivalent to 258 bits in length. Therefore, the key space is large enough to resist brute-force attack.

\subsection{Statistical Analysis}

\subsubsection{Histogram Analysis}

A histogram of an image demonstrates the distribution of the image pixel values, and it exposes the pixel distribution characteristics of the image. The more uniform the distribution of the pixel values, the closer the image is to the random signal image. Figure 7 shows the histograms of the above test plain images and cipher images encrypted by our proposed algorithm (the histograms of the all-white and all-black plain images are omitted). It can be seen from Figure 7 that the distributions of pixel values in plain images are clearly not uniform but in cipher images are very uniform. 


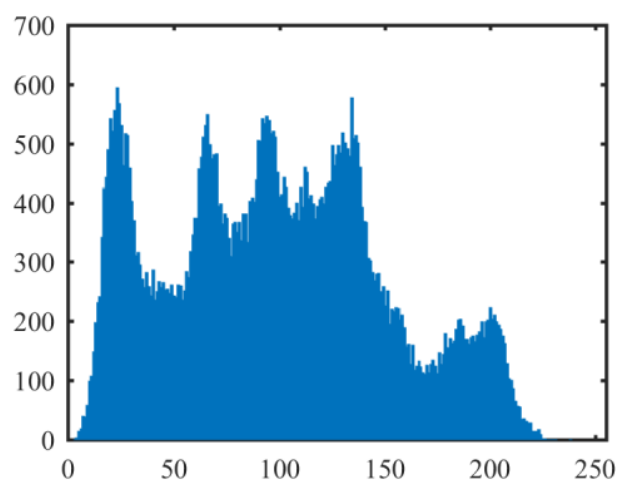

(a)

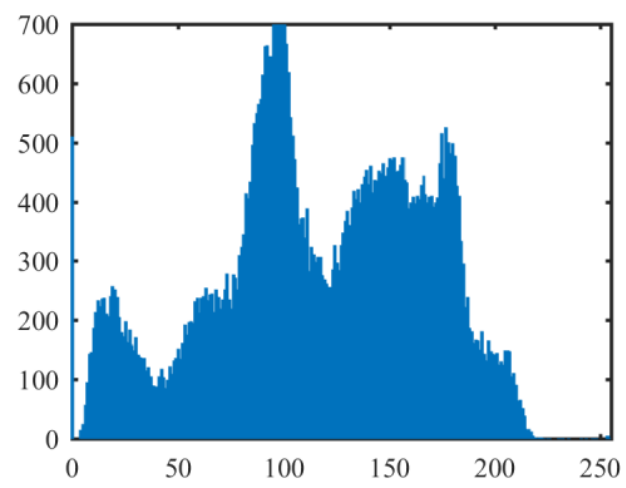

(c)

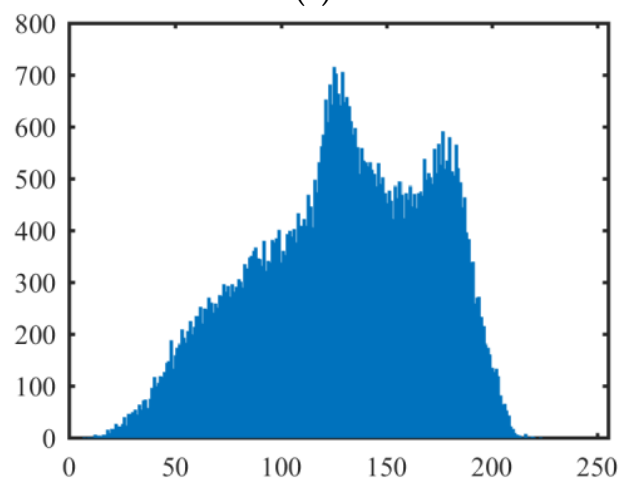

(e)

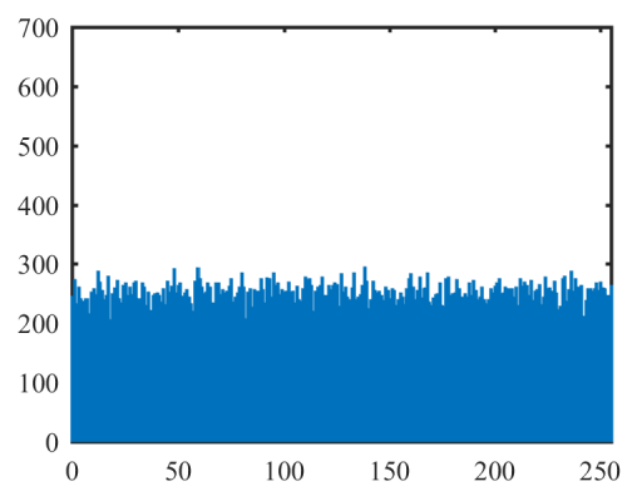

(g)

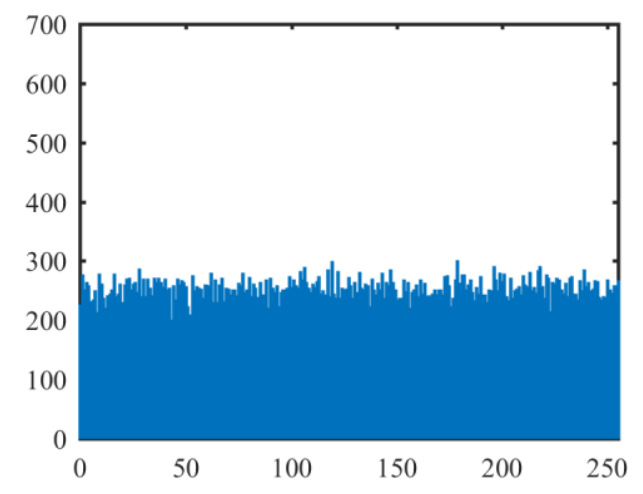

(b)

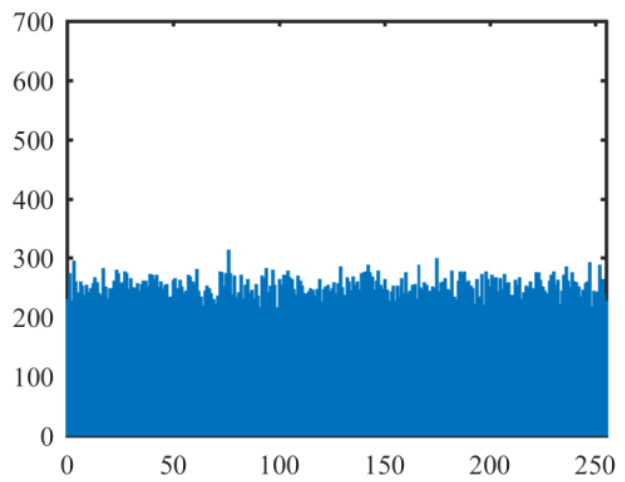

(d)

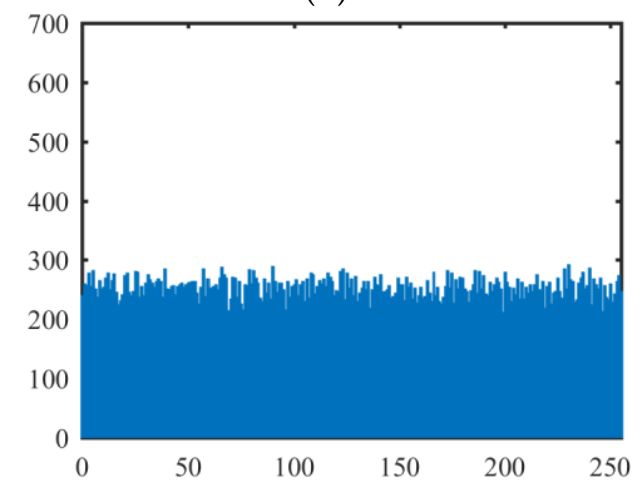

(f)

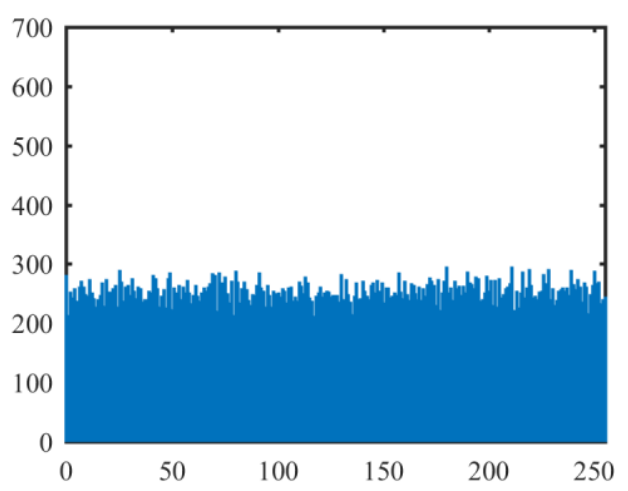

(h)

Figure 7. Histograms of plain images and cipher images. (a) The histogram of the Lena plain image; (b) the histogram of the Lena cipher image; (c) the histogram of the Peppers plain image; (d) the histogram of the Peppers cipher image; (e) the histogram of the Baboon plain image; (f) the histogram of the Baboon cipher image; (g) the histogram of the all-white cipher image; (h) the histogram of the all-black cipher image. 
The distribution characteristics of a histogram can also be described quantitatively with the variance of a histogram, which is calculated by [16]

$$
\operatorname{var}(\mathbf{Z})=\frac{1}{n^{2}} \sum_{i=1}^{n} \sum_{j=1}^{n} \frac{1}{2}\left(z_{i}-z_{j}\right)^{2}
$$

where, $n$ is the number of gray levels of an image, and $n=256$ for 8-bit gray images. $\mathbf{Z}$ is a vector and $\mathbf{Z}=\left\{z_{1}, z_{2}, \ldots, z_{\mathrm{n}}\right\}, z_{i}$ and $z_{j}$ are the numbers of pixels with gray values equal to $(i-1)$ and $(j-1)$ respectively. The lower value of variance indicates the higher uniformity of an image. In order to detect the variance values of the above test images and their cipher images, the variances of histograms of the plain images (size of $256 \times 256$ ) and their cipher images are calculated by using Equation (16). The results are listed in Table 4. Table 4 also lists the results obtained by the algorithm in References [39] and [40]. The average variance of five cipher images obtained with our proposed algorithm is 256.7125, which is much less than that of Zhang's algorithm [39], Wang's algorithm [40], and Çavuşoğlu's algorithm [57]. Thus, our proposed image encryption algorithm has better performance in resisting statistical attacks.

Table 4. Variances of histograms of the test images.

\begin{tabular}{cccccc}
\hline Images & Plain Image & $\begin{array}{l}\text { Cipher } \\
\text { Image }\end{array}$ & Cipher Image [39] & Cipher Image [40] & Cipher Image [57] \\
\hline Lena & $30,665.703$ & 221.195 & 284.578 & 283.156 & 381.688 \\
Peppers & $36,379.133$ & 224.234 & 269.727 & 227.898 & 332.898 \\
Baboon & $47,799.055$ & 288.664 & 268.211 & 277.297 & 297.625 \\
All-white image & $16,711,680$ & 293.039 & 544.234 & $41,725.063$ & 1214.484 \\
All-black image & $16,711,680$ & 256.430 & 1396.765 & $43,233.188$ & 1214.484 \\
cipher image & $6,707,640.778$ & 256.713 & 552.703 & $17,149.320$ & 688.236 \\
Average & & & & & \\
\hline
\end{tabular}

\subsubsection{Correlation Analysis}

Natural images usually have a strong correlation with adjacent pixels. An efficient encryption algorithm should reduce the correlation in cipher images. In order to exhibit the correlation strength intuitively, we randomly selected 2000 pairs of pixel along a certain direction (horizontal or vertical or diagonal) from an image to draw the correlation distribution diagram. Figure 8 shows the correlation distribution diagrams of the Lena plain and cipher image encrypted by our encryption algorithm. The abscissa and ordinate values at any point in the graph represent the values of a pair of neighbor pixels, respectively. For plaintext images, most of the points in the graph are distributed near a straight line with an inclination of 45 degrees. That is to say, the abscissa and ordinate coordinates of most points are basically equal, indicating that the pixel values of neighboring points in plaintext images are basically equal. However, the pixel values of each group of neighbor points in ciphertext images are not equal. The results confirm that the correlation among the adjacent pixels is reduced greatly by our proposed encryption algorithm.

To illustrate quantitatively the correlation of adjacent pixels in an image, we can calculate the correlation coefficient $r_{X Y}$ by using $N$ pairs of an adjacent pixel. $r_{X Y}$ is defined as

$$
r_{X Y}=\operatorname{cov}(X, Y) / \sqrt{D(X)} \sqrt{D(Y)}
$$

where, $\mathrm{X}=\left\{x_{1}, x_{2}, \ldots, x_{\mathrm{N}}\right\}$ and $\mathrm{Y}=\left\{y_{1}, y_{2}, \ldots, y_{N}\right\},\left(x_{i}, y_{i}\right)$ is the $i$-th pairs of the adjacent pixel gray-scale values, and

$$
D(X)=\frac{1}{N} \sum_{i=1}^{N}\left(x_{i}-\bar{X}\right)^{2}, D(Y)=\frac{1}{N} \sum_{i=1}^{N}\left(x_{i}-\bar{Y}\right)^{2}
$$




$$
\begin{gathered}
\operatorname{cov}(X, Y)=\frac{1}{N} \sum_{i=1}^{N}\left(x_{i}-\bar{X}\right)\left(y_{i}-\bar{Y}\right) \\
\bar{X}=\frac{1}{N} \sum_{i=1}^{N} x_{i}, \bar{Y}=\frac{1}{N} \sum_{i=1}^{N} y_{i}
\end{gathered}
$$

Three types of correlation coefficients of adjacent pixels in the Lena plain and cipher image are calculated, respectively. Correlation coefficients of the Lena plain images are as: 0.9567 (horizontal direction), 0.9239 (vertical direction), 0.8888 (diagonal direction), showing that correlation coefficients of adjacent pixels in the Lena plain image are very high (all close to one). Results of the Lena cipher image are listed in Table 5. From Table 5, we can see that the correlation coefficients of adjacent pixels in the Lena cipher image are very low (all close to zero). Table 5 also lists the correlation coefficients of the Lena cipher image encrypted with Zhang's algorithm, Wang's algorithm and Çavuşoğlu's algorithm. The experimental results show that our proposed algorithm has the smallest absolute values of the correlation coefficient among the three algorithms, showing the best scrambling effect.

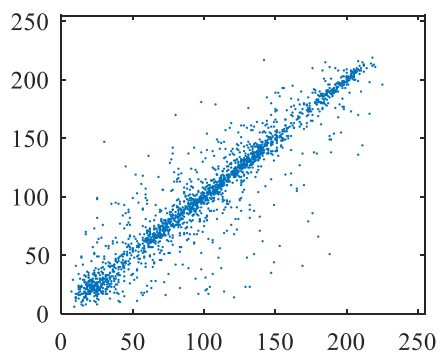

(a)

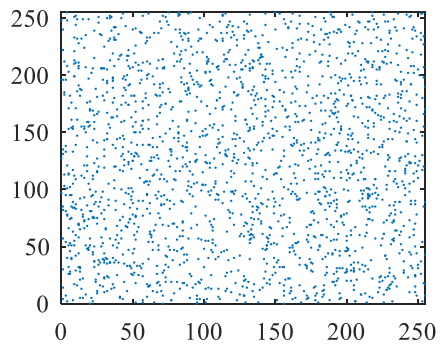

(d)

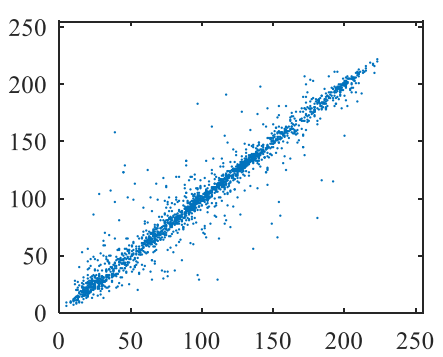

(b)

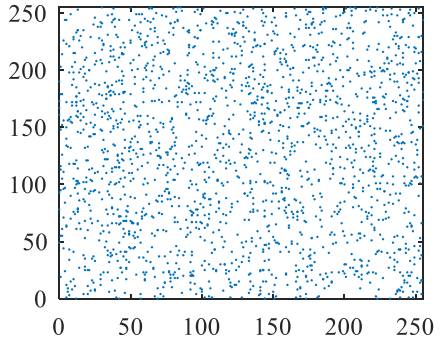

(e)

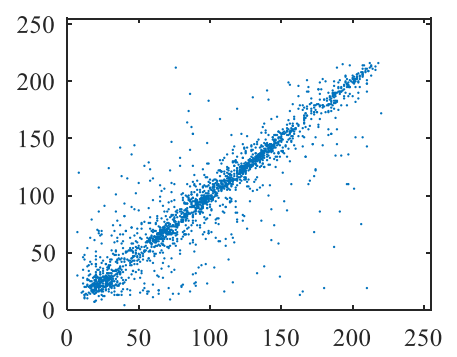

(c)

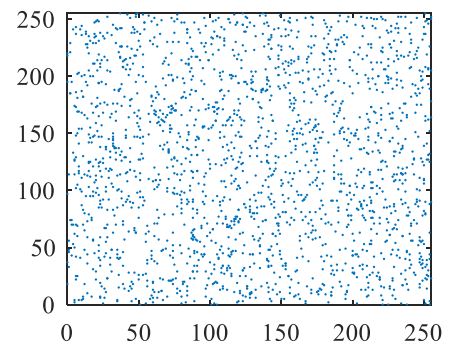

(f)

Figure 8. Correlation analysis of the plain and cipher Lena. (a) Horizontal correlation in plain image Lena; (b) vertical correlation in plain image Lena; (c) diagonal correlation in plain image Lena; (d) horizontal correlation in cipher image Lena; (e) vertical correlation in cipher image Lena; (f) diagonal correlation in cipher image Lena.

Table 5. Correlation coefficients of the Lena cipher images encrypted by different algorithms.

\begin{tabular}{cccc}
\hline Algorithms & Horizontal & Vertical & Diagonal \\
\hline The proposed algorithm & -0.002088 & 0.000312 & 0.001444 \\
Zhang's algorithm [39] & -0.000582 & 0.001336 & -0.004690 \\
Wang's algorithm [40] & 0.006057 & 0.012468 & -0.006030 \\
Çavuşoğlu's algorithm [57] & 0.001640 & 0.031372 & -0.000626 \\
\hline
\end{tabular}




\subsubsection{Information Entropy Analysis}

Information entropy can be used to describe the degree of randomness or uncertainty of signals. The information entropy $H(m)$ of an image is calculated by

$$
H(m)=-\sum_{i=0}^{2^{n}-1} P\left(m_{i}\right) \log _{2}\left[P\left(m_{i}\right)\right]
$$

where $P\left(m_{i}\right)$ denotes the occurrence probability of the gray level $i$, and $i=0,1,2, \ldots, 2^{n}$. Here, $2^{n}$ is the number of grayscale levels of an image. If each $m_{i}$ has the same occurrence probability in an image, then $P\left(m_{i}\right)=1 / 2^{n}$, then the image is completely random with $H(m)=n$. For an image with 256 gray-scale levels, $n=8$, so, the information entropy of a completely random 8-bit gray image is eight. A good encryption algorithm should make the information entropy of its cipher image close to eight. We calculated the information entropy values of several cipher images obtained by four different encryption algorithms. The results are listed in Table 6. All the images have the same size of $256 \times 256$. From Table 6, one can see that all the entropy values are significantly closer to eight, so the randomness is satisfactory. Among these four algorithms, our proposed algorithm has the largest average entropy value, showing the best randomness of the cipher image encrypted by our proposed algorithm.

Table 6. Information entropy values of several cipher images obtained by different algorithms.

\begin{tabular}{ccccc}
\hline Test Images & Ref. [39] & Ref. [40] & Ref. [57] & Ours \\
\hline Lena cipher image & 7.9969 & 7.9969 & 7.9958 & 7.9976 \\
Peppers cipher image & 7.9970 & 7.9975 & 7.9963 & 7.9975 \\
Baboon cipher image & 7.9970 & 7.9969 & 7.9967 & 7.9968 \\
All-black cipher image & 7.9846 & 7.3901 & 7.9871 & 7.9972 \\
All-white cipher image & 7.9940 & 7.3998 & 7.9871 & 7.9968 \\
\hline
\end{tabular}

\subsubsection{Sensitivity Analysis}

\section{(1) Sensitivity to plain images}

A secure encryption algorithm should be sensitive to the change of the plain image so as to resist the differential attack. To measure the sensitivity of an algorithm to tiny changes in a plain image, the number of pixels changing rate (NPCR) and the unified average changing intensity (UACI) are introduced. The NPCR and UACI are calculated by Equations (22)-(24).

$$
\begin{gathered}
\mathrm{NPCR}=\frac{1}{M \times N} \sum_{i=1}^{M} \sum_{j=1}^{N} \delta(i, j) \times 100 \% \\
\mathrm{UACI}=\frac{1}{M \times N}\left(\sum_{i=1}^{M} \sum_{j=1}^{N} \frac{\left|c_{1}(i, j)-c_{2}(i, j)\right|}{255}\right) \times 100 \%
\end{gathered}
$$

where, $M, N$ represent the number of rows and columns of an image, respectively. $\mathbf{C}_{1}=\left[c_{1}(i, j)\right]$ and $\mathbf{C}_{2}=\left[c_{2}(i, j)\right]$ express two encrypted images corresponding to two plain images with a tiny difference, and $\delta(i, j)$ is computed by

$$
\delta(i, j)= \begin{cases}1, & \text { if } c_{1}(i, j) \neq c_{2}(i, j) \\ 0, & \text { if } c_{1}(i, j)=c_{2}(i, j) .\end{cases}
$$

The larger the values of NPCR and UACI, the stronger the sensitivity of the algorithm to plaintext. For the best case, the ideal average value of NPCR is about $99.61 \%$, and the ideal average value of UACI is about $33.46 \%$ [16].

To measure the sensitivity of our improved algorithm to the plain image, the original Lena gray image (size of $256 \times 256$ ) is adopted as the first plain image, and the second plain image is obtained by 
changing only one pixel of the first plain image. To obtain two cipher images $\mathbf{C}_{1}$ and $\mathbf{C}_{2}$ by executing the proposed encryption algorithm with the same secret keys, respectively. Then NPCR and UACI are computed with two cipher images, and the results are listed in Table 7 . Table 7 also lists the results obtained by using the Zhang's, Wang's and Çavuşoğlu's algorithm. The results indicate that our proposed encryption algorithm is very sensitive to the plain image, and its sensitivity is better than those of Zhang's and Wang's algorithm.

Table 7. Values of number of pixels changing rate (NPCR) and unified average changing intensity (UACI) of Lena cipher images.

\begin{tabular}{cccccc}
\hline Position $i$ & Values & Zhang's [39] & Wang's [40] & Çavuşoğlu's [57] & Ours \\
\hline 1 & NPCR(\%) & 49.81 & $1.53 \times 10^{-3}$ & $1.53 \times 10^{-3}$ & 99.64 \\
1 & UACI(\%) & 16.86 & $1.14 \times 10^{-3}$ & $2.75 \times 10^{-4}$ & 33.55 \\
$L / 4$ & NPCR(\%) & 74.69 & $1.53 \times 10^{-3}$ & $1.53 \times 10^{-3}$ & 99.59 \\
$L / 4$ & UACI(\%) & 25.08 & $1.68 \times 10^{-4}$ & $8.26 \times 10^{-4}$ & 33.25 \\
$L / 2$ & NPCR(\%) & 99.64 & $1.53 \times 10^{-3}$ & $1.53 \times 10^{-3}$ & 99.57 \\
$L / 2$ & UACI(\%) & 33.54 & $6.10 \times 10^{-4}$ & $4.13 \times 10^{-4}$ & 33.41 \\
$L$ & NPCR(\%) & 49.84 & $1.53 \times 10^{-3}$ & $1.53 \times 10^{-3}$ & 99.62 \\
$L$ & UACI(\%) & 16.72 & $8.80 \times 10^{-4}$ & $8.62 \times 10^{-4}$ & 33.46 \\
\hline
\end{tabular}

\section{(2) Sensitivity to Secret Keys}

A secure encryption algorithm should also be sensitive to the change of secret keys. That is to say, when secret keys change slightly, the cipher image should change dramatically. NPCR and UACI can also be used to measure the sensitivity of an encryption algorithm to secret keys. In our simulation tests, two groups of secret keys with a tiny difference are used to encrypt the same plain image Lena and two cipher images, $\mathbf{C}_{1}$ and $\mathbf{C}_{2}$, are obtained. The tiny change (to a float number is $10^{-15}$, or to an integer number is one) is introduced to one of the secret keys $\left(x_{10}, \mu_{1}, d_{1}, x_{20}, \mu_{2}, d_{2}, r_{0}, t_{0}, m\right)$ while keeping all the others unchanged. The NPCR and UACI of the cipher images $\mathbf{C}_{1}$ and $\mathbf{C}_{2}$ are calculated and listed in Table 8 . The experimental results indicate that our proposed algorithm is very sensitive to a slight change in any secret key.

Table 8. NPCR and UACI of the proposed algorithm with a tiny difference in one of the secret keys.

\begin{tabular}{|c|c|c|c|c|c|c|c|c|c|}
\hline Values & $\Delta x_{10}=10^{-15}$ & $\Delta \mu_{1}=10^{-15}$ & $\Delta x_{20}=10^{-15}$ & $\Delta \mu_{2}=10^{-15}$ & $\Delta d_{1}=1$ & $\Delta d_{2}=1$ & $\Delta r_{0}=1$ & $\Delta t_{0}=1$ & $\Delta m=1$ \\
\hline NPCR(\%) & 99.63 & 99.62 & 99.56 & 99.62 & 99.61 & 99.58 & 99.63 & 99.61 & 99.61 \\
\hline UACI $(\%)$ & 33.53 & 33.34 & 33.50 & 33.41 & 33.38 & 33.53 & 33.46 & 33.41 & 33.37 \\
\hline
\end{tabular}

\subsection{Analysis of Anti-Attack Performance}

\subsubsection{Classical Types of Attacks}

According to Kerchoff's hypothesis, it is usually assumed that the cryptanalysts or opponents know the cryptosystem, and the security entirely depends on the secret key. A secure cryptosystem should resist all kinds of attacks; otherwise, the cryptosystem is insecure. Generally speaking, there are four classical types of attacks to break a cryptosystem, and their orders from the hardest types to the easiest types are listed as follows.

(1) Ciphertext-only attack: The cryptanalyst possesses one or more ciphertexts.

(2) Known-plaintext attack: The cryptanalyst has some plaintexts and the corresponding ciphertexts.

(3) Chosen-plaintext attack: The cryptanalyst has the opportunity to use the encryption machinery, so he or she can choose some plaintext and generate ciphertext.

(4) Chosen-ciphertext attack: The cryptanalyst has the opportunity to use the cryptograph, so he or she can choose some ciphertexts and generate plaintexts. 
Among the four classical attack types mentioned above, the chosen-ciphertext attack is the most powerful attack. If a cryptosystem can resist this attack, it can resist other types of attacks.

In our proposed scheme, $\{\mathbf{S 1}, \mathbf{S} \mathbf{2}, \mathbf{r}, \mathbf{t}\}$ become the equivalent keys to the original keys. It is not difficult to understand the following conclusions from the encryption formulas of Equations (8)-(11). First, it is difficult for an attacker to decipher the above equivalent keys even if he or she obtains known plaintext-ciphertext pairs $(p(i), c(i))$. Second, the equivalent keys $\mathbf{r}$ and $\mathbf{t}$ are updated before encrypting the $i$-th pixel and they are related with the intermediate ciphertext $b(i-1)$ or the final ciphertext $c(i+1)$. It means that a different cipher image will yield different sequences of $\{\mathbf{r}, \mathbf{t}\}$. Even if the attacker cracked the key sequences of $\{\mathbf{r}, \mathbf{t}\}$ with some specially chosen-ciphertext, the key streams of $\{\mathbf{r}, \mathbf{t}\}$ cannot be used to decrypt the target cipher image due to the key streams of the target cipher image that are different from the cracked key streams. Moreover, it is difficult to decipher the key streams $\{\mathbf{r}, \mathbf{t}\}$ directly by using the chosen-ciphertext attack. Therefore, the proposed scheme can well resist the chosen-ciphertext attack and can resist the four classical types of attacks.

\subsubsection{Analysis of Robustness against Noise and Occlusion}

In order to resist the differential cryptanalysis attack brought by the opponent, a strong diffusion mechanism is introduced into the proposed encryption algorithm. As a result, the ciphertext is sensitive to the noise of the transmission channel, so the algorithm lacks robustness to noise and occlusion. However, the lack of such robustness also makes it impossible for the opponent to decipher the plaintext accurately, which can ensure that the confidentiality of the image content is protected. As for how to make the encrypted image not only resist differential attack, but also withstand a certain degree of noise, we consider introducing an error correction mechanism in channel coding and decoding. This is worthy of further study in the future.

\subsection{Analysis of Speed}

In addition to security performance, a practical cryptosystem should also have faster encryption speed. To evaluate the encryption efficiency of the proposed algorithm, the 8-bit greyscale images with a size of $256 \times 256$ and $512 \times 512$ are encrypted. And the same type of S-box based image encryption algorithms proposed by Zhang [39], Wang [40], and Çavuşoğlu [57] are also implemented on the same hardware and software platform mentioned at the beginning of Section 5 . The average values of the encryption/decryption time taken by Zhang's algorithm, Wang's algorithm, Çavuşoğlu's algorithm and our proposed algorithm are shown in Table 9, respectively. The experimental results show the advantages of the proposed algorithm in time efficiency.

Table 9. The time cost tests (unit: s).

\begin{tabular}{ccccc}
\hline Image Size & Ref. [39] & Ref. [40] & Ref. [57] & This Paper \\
\hline $256 \times 256$ & 1.205 & 1.256 & 0.823 & 0.464 \\
$512 \times 512$ & 4.750 & 4.828 & 3.253 & 1.708 \\
\hline
\end{tabular}

Our proposed algorithm has an execution time that includes: Two S-boxes generated by a novel simple method using the $1 \mathrm{D}$ discrete chaotic map, $2 L$ times of byte substitution and $2 L$ times mod 256 addition operations. Zhang's algorithm execution time include: Two S-boxes generated by an ordinary method using the $1 \mathrm{D}$ discrete chaotic map, $2 L$ times of byte substitution, $L$ times mod 256 addition operations and $L$ times bitXor operations. Wang's algorithm has an execution time that includes: Three S-boxes generated by an ordinary method using the 3D continuous-time chaotic system, $L$ times of byte substitution, $L$ times mod 3 addition operations and $L$ times bitXor operations. Çavuşoğlu's algorithm has an execution time that includes: One S-box generated by an ordinary method using the $3 \mathrm{D}$ continuous-time chaotic system, $L$ times of byte substitution and $L$ times bitXor operations. The mod addition operation has a less execution time than the bitXor operation, and the bitXor operation has a 
less execution time than the byte substitution operation. Our algorithm to generate the S-box has the least execution time among the four algorithms. As the result, the total execution time of our algorithm is the smallest one among the four algorithms.

\section{Conclusions}

In this paper, an efficient and secure image encryption scheme is presented. The main contributions of this paper are as follows: First, a new compound chaotic system, the Sine-Tent map, is proposed, which has wider chaotic range and better chaotic performance than any of the old one. And the new compound chaotic system is more suitable for cryptosystem. Second, an efficient and secure method for generating S-boxes is proposed, which has less execution time than the other ones. Third, a novel double S-boxes based image encryption algorithm is proposed. By introducing equivalent key sequences $\{\mathbf{r}, \mathbf{t}\}$ related with image ciphertext, the proposed cryptosystem can resist the four classical types of attacks, which is an advantage over other S-box based encryption schemes. It overcomes the security defects of some old S-box based encryption algorithms. In addition, two rounds of forward and backward confusion-diffusion operation enhance the sensitivity of the algorithm. The simulation results and security analysis verify the effectiveness of the proposed scheme. The new scheme has obvious efficiency advantages, which means that it has better application potential in real-time image encryption. The proposed scheme is also suitable to color images by connecting three color channels of color image into gray image.

As for the research of the chaotic image encryption, there are two aspects worthy of further study in the future. First, we need to explore new security evaluation criteria to make up for the shortcomings of empirical security standards. Second, in order to ensure that the encryption system is not only resistant to differential cryptanalysis attacks, but also robust to noise, it may be an effective solution to introduce error-correcting codes in the process of cryptography and decoding.

Author Contributions: Conceptualization, C.Z. and S.Z.; Methodology, G.W.; Software, S.Z.; Validation, C.Z., S.Z. and G.W.; Formal analysis, C.Z.; Investigation, C.Z.; Resources, C.Z.; Data curation, S.Z.; Writing-Original draft preparation, S.Z.; Writing-Review and editing, C.Z. and G.W.; Visualization, C.Z.; Supervision, G.W.; Project administration, C.Z.; Funding acquisition, G.W.

Funding: This research was funded by [the National Natural Science Foundation of China] grant number [No. 61632009] and [Guangdong Provincial Natural Science Foundation] grant number [No. 2017A030308006].

Acknowledgments: The authors are thankful to the reviewers for their comments and suggestions to improve the quality of the manuscript.

Conflicts of Interest: The authors declare no conflict of interest.

\section{References}

1. Wang, J.; Ding, Q. Dynamic rounds chaotic block cipher based on keyword abstract extraction. Entropy 2018, 20, 693. [CrossRef]

2. Abdallah, E.E.; Ben Hamza, A.; Bhattacharya, P. Mpeg video watermarking using tensor singular value decomposition. In Lecture Notes in Computer Science, Proceedings of the Image Analysis and Recognition (ICIAR 2007), Montreal, QC, Canada, 5-7 July 2017; Kamel, M., Campilho, A., Eds.; Springer: Berlin/Heidelberg, Germany, 2007; Volume 4633, pp. 772-783.

3. Abdallah, E.E.; Ben Hamza, A.; Bhattacharya, P. Video watermarking using wavelet transform and tensor algebra. Signal Image Video Process. 2009, 4, 233-245. [CrossRef]

4. Zhang, S.; Li, X.; Tan, Z.; Peng, T.; Wang, G. A caching and spatial k-anonymity driven privacy enhancement scheme in continuous location-based services. Future Gener. Comput. Syst. 2019, 94, 40-50. [CrossRef]

5. Zhang, S.; Wang, G.; Bhuiyan, M.Z.A.; Liu, Q. A dual privacy preserving scheme in continuous location-based services. IEEE Internet Things J. 2018, 5, 4191-4200. [CrossRef]

6. Wang, X.; Pham, V.-T.; Jafari, S.; Volos, C.; Munoz-Pacheco, J.M.; Tlelo-Cuautle, E. A new chaotic system with stable equilibrium: From theoretical model to circuit implementation. IEEE Access 2017, 5, 8851-8858. [CrossRef]

7. Zhou, Y.; Bao, L.; Chen, C.L.P. A new 1D chaotic system for image encryption. Signal Process. 2014, 97, $172-182$. [CrossRef] 
8. Chen, E.; Min, L.; Chen, G. Discrete chaotic systems with one-line equilibria and their application to image encryption. Int. J. Bifurc. Chaos 2017, 27, 1750046. [CrossRef]

9. Zhu, S.; Zhu, C.; Cui, H.; Wang, W. A class of quadratic polynomial chaotic maps and its application in cryptography. IEEE Access 2019, 7, 34141-34152. [CrossRef]

10. Sahari, M.L.; Boukemara, I. A pseudo-random numbers generator based on a novel 3d chaotic map with an application to color image encryption. Nonlinear Dyn. 2018, 94, 723-744. [CrossRef]

11. Murillo-Escobar, M.A.; Cruz-Hernandez, C.; Cardoza-Avendano, L.; Mendez-Ramirez, R. A novel pseudorandom number generator based on pseudorandomly enhanced logistic map. Nonlinear Dyn. 2017, 87, 407-425. [CrossRef]

12. Islam, F.u.; Liu, G. Designing s-box based on 4D-4wing hyperchaotic system. 3D Res. 2017, 8, 9. [CrossRef]

13. Alvarez, G.; Li, S. Some basic cryptographic requirements for chaos-based cryptosystems. Int. J. Bifurc. Chaos 2006, 16, 2129-2151. [CrossRef]

14. Li, C.; Lin, D.; Feng, B.; Lu, J.; Hao, F. Cryptanalysis of a chaotic image encryption algorithm based on information entropy. IEEE Access 2018, 6, 75834-75842. [CrossRef]

15. Zhu, C.; Wang, G.; Sun, K. Improved cryptanalysis and enhancements of an image encryption scheme using combined 1d chaotic maps. Entropy 2018, 20, 843. [CrossRef]

16. Zhu, C.; Sun, K. Cryptanalyzing and improving a novel color image encryption algorithm using rt-enhanced chaotic tent maps. IEEE Access 2018, 6, 18759-18770. [CrossRef]

17. Fridrich, J. Symmetric ciphers based on two-dimensional chaotic maps. Int. J. Bifurc. Chaos 1998, 8, 1259-1284. [CrossRef]

18. Zhang, X.; Fan, X.; Wang, J.; Zhao, Z. A chaos-based image encryption scheme using 2D rectangular transform and dependent substitution. Multimed. Tools Appl. 2014, 75, 1745-1763. [CrossRef]

19. Zhang, Y.; Xiao, D. Double optical image encryption using discrete chirikov standard map and chaos-based fractional random transform. Opt. Lasers Eng. 2013, 51, 472-480. [CrossRef]

20. Gan, Z.-h.; Chai, X.-1.; Han, D.-j.; Chen, Y.-r. A chaotic image encryption algorithm based on 3-d bit-plane permutation. Neural Comput. Appl. 2018, 2018, 1-20. [CrossRef]

21. Hu, G.; Xiao, D.; Zhang, Y.; Xiang, T. An efficient chaotic image cipher with dynamic lookup table driven bit-level permutation strategy. Nonlinear Dyn. 2016, 87, 1359-1375. [CrossRef]

22. Ye, G.; Zhao, H.; Chai, H. Chaotic image encryption algorithm using wave-line permutation and block diffusion. Nonlinear Dyn. 2016, 83, 2067-2077. [CrossRef]

23. Abd-El-Hafiz, S.K.; AbdElHaleem, S.H.; Radwan, A.G. Novel permutation measures for image encryption algorithms. Opt. Lasers Eng. 2016, 85, 72-83. [CrossRef]

24. Li, Y.; Wang, C.; Chen, H. A hyper-chaos-based image encryption algorithm using pixel-level permutation and bit-level permutation. Opt. Lasers Eng. 2017, 90, 238-246. [CrossRef]

25. Zhang, Y.; Xiao, D.; Shu, Y.; Li, J. A novel image encryption scheme based on a linear hyperbolic chaotic system of partial differential equations. Signal Process. Image Commun. 2013, 28, 292-300. [CrossRef]

26. Wang, X.; Liu, C.; Zhang, H. An effective and fast image encryption algorithm based on chaos and interweaving of ranks. Nonlinear Dyn. 2016, 84, 1595-1607. [CrossRef]

27. Xu, L.; Gou, X.; Li, Z.; Li, J. A novel chaotic image encryption algorithm using block scrambling and dynamic index based diffusion. Opt. Lasers Eng. 2017, 91, 41-52. [CrossRef]

28. Hua, Z.; Yi, S.; Zhou, Y. Medical image encryption using high-speed scrambling and pixel adaptive diffusion. Signal Process. 2018, 144, 134-144. [CrossRef]

29. Huang, H.; He, X.; Xiang, Y.; Wen, W.; Zhang, Y. A compression-diffusion-permutation strategy for securing image. Signal Process. 2018, 150, 183-190. [CrossRef]

30. Cao, C.; Sun, K.; Liu, W. A novel bit-level image encryption algorithm based on 2D-LICM hyperchaotic map. Signal Process. 2018, 143, 122-133. [CrossRef]

31. Chai, X. An image encryption algorithm based on bit level brownian motion and new chaotic systems. Multimed. Tools Appl. 2017, 76, 1159-1175. [CrossRef]

32. Hua, Z.; Jin, F.; Xu, B.; Huang, H. 2D Logistic-Sine-coupling map for image encryption. Signal Process. 2018, 149, 148-161. [CrossRef]

33. Hua, Z.; Zhou, Y. Image encryption using 2D Logistic-adjusted-Sine map. Inf. Sci. 2016, 339, 237-253. [CrossRef]

34. Kaur, M.; Kumar, V. Efficient image encryption method based on improved Lorenz chaotic system. Electron. Lett. 2018, 54, 562-564. [CrossRef] 
35. Liu, J.; Yang, D.; Zhou, H.; Chen, S. A digital image encryption algorithm based on bit-planes and an improved Logistic map. Multimed. Tools Appl. 2018, 77, 10217-10233. [CrossRef]

36. Zhu, C. A novel image encryption scheme based on improved hyperchaotic sequences. Opt. Commun. 2012, 285, 29-37. [CrossRef]

37. Zhang, Y. The image encryption algorithm based on chaos and DNA computing. Multimed. Tools Appl. 2018, 77, 21589-21615. [CrossRef]

38. Farwa, S.; Shahy, T.; Muhammad, N.; Bibiz, N.; Jahangir, A.; Arshad, S. An image encryption technique based on chaotic S-box and Arnold transform. Int. J. Adv. Comput. Sci. Appl. 2017, 8, 360-364. [CrossRef]

39. Zhang, X.-P.; Guo, R.; Chen, H.-W.; Zhao, Z.-M.; Wang, J.-Y. Efficient image encryption scheme with synchronous substitution and diffusion based on double S-boxes. Chin. Phys. B 2018, 27, 080701. [CrossRef]

40. Wang, X.; Çavuşoğlu, Ü.; Kacar, S.; Akgul, A.; Pham, V.-T.; Jafari, S.; Alsaadi, F.; Nguyen, X. S-box based image encryption application using a chaotic system without equilibrium. Appl. Sci. 2019, 9, 781. [CrossRef]

41. Zhu, S.; Zhu, C.; Wang, W. A new image encryption algorithm based on chaos and secure hash SHA-256. Entropy 2018, 20, 716. [CrossRef]

42. Zhu, S.; Zhu, C.; Wang, W. A novel image compression-encryption scheme based on chaos and compression sensing. IEEE Access 2018, 6, 67095-67107. [CrossRef]

43. Zhu, S.; Zhu, C. Image encryption algorithm with an avalanche effect based on a six-dimensional discrete chaotic system. Multimed. Tools Appl. 2018, 77, 29119-29142. [CrossRef]

44. Sun, S.; Guo, Y.; Wu, R. A novel image encryption scheme based on 7D hyperchaotic system and row-column simultaneous swapping. IEEE Access 2019, 7, 28539-28547. [CrossRef]

45. Zhang, S.; Wang, G.; Liu, Q.; Abawajy, J.H. A trajectory privacy-preserving scheme based on query exchange in mobile social networks. Soft Comput. 2018, 22, 6121-6133. [CrossRef]

46. Bhuiyan, M.Z.A.; Wang, G.; Wu, J.; Cao, J.; Liu, X.; Wang, T. Dependable structural health monitoring using wireless sensor networks. IEEE Trans. Dependable Secur. Comput. 2017, 14, 363-376. [CrossRef]

47. Zhang, Q.; Liu, Q.; Wang, G. PRMS: A personalized mobile search over encrypted outsourced data. IEEE Access 2018, 6, 31541-31552. [CrossRef]

48. Sun, K.-H.; He, S.-B.; Yin, L.-Z.; Li-Kun, A.D.-L.D. Application of fuzzyen algorithm to the analysis of complexity of chaotic sequence. Acta Phys. Sin. 2012, 61, 130507.

49. Sun, K.-H.; He, S.-B.; He, Y.; Yin, L.-Z. Complexity analysis of chaotic pseudo-random sequences based on spectral entropy algorithm. Acta Phys. Sin. 2013, 62, 010501.

50. He, S.-B.; Sun, K.-H.; Zhu, C.-X. Complexity analyses of multi-wing chaotic systems. Chin. Phys. B 2013, 22, 050506. [CrossRef]

51. Khan, M. A novel image encryption scheme based on multiple chaotic S-boxes. Nonlinear Dyn. 2015, 82, 527-533. [CrossRef]

52. Wang, X.; Wang, Q. A novel image encryption algorithm based on dynamic S-boxes constructed by chaos. Nonlinear Dyn. 2013, 75, 567-576. [CrossRef]

53. Murillo-Escobar, M.A.; Cruz-Hernández, C.; Abundiz-Pérez, F.; López-Gutiérrez, R.M.; Acosta Del Campo, O.R. A rgb image encryption algorithm based on total plain image characteristics and chaos. Signal Process. 2015, 109, 119-131. [CrossRef]

54. Zhang, Y.; Tang, Y. A plaintext-related image encryption algorithm based on chaos. Multimed. Tools Appl. 2017, 77, 6647-6669. [CrossRef]

55. Preishuber, M.; Hutter, T.; Katzenbeisser, S.; Uhl, A. Depreciating motivation and empirical security analysis of chaos-based image and video encryption. IEEE Trans. Inf. Forensics Secur. 2018, 13, 2137-2150. [CrossRef]

56. Belazi, A.; El-Latif, A.A.A. A simple yet efficient S-box method based on chaotic Sine map. Optik 2017, 130, 1438-1444. [CrossRef]

57. Çavuşoğlu, Ü.; Kaçar, S.; Pehlivan, I.; Zengin, A. Secure image encryption algorithm design using a novel chaos based S-box. Chaos Solitons Fractals 2017, 95, 92-101. [CrossRef]

58. Zhu, C.X.; Wang, G.J.; Sun, K.H. Cryptanalysis and improvement on an image encryption algorithm design using a novel chaos based S-box. Symmetry 2018, 10, 399. [CrossRef]

(C) 2019 by the authors. Licensee MDPI, Basel, Switzerland. This article is an open access article distributed under the terms and conditions of the Creative Commons Attribution (CC BY) license (http://creativecommons.org/licenses/by/4.0/). 